\title{
Oral sucrosomial iron versus intravenous iron in anemic cancer patients without iron deficiency receiving darbepoetin alfa: a pilot study
}

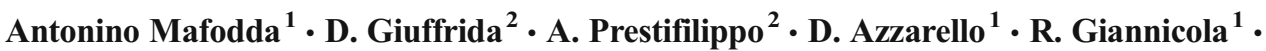 \\ M. Mare $^{2} \cdot$ R. Maisano ${ }^{1}$
}

Received: 19 August 2016 / Accepted: 31 March 2017 /Published online: 9 April 2017

(C) The Author(s) 2017. This article is published with open access at Springerlink.com

\begin{abstract}
Purpose Erythropoiesis-stimulating agents (ESAs) are often used in treatment of patients with chemotherapy-induced anemia. Many studies have demonstrated an improved hemoglobin $(\mathrm{Hb})$ response when ESA is combined with intravenous iron supplementation and a higher effectiveness of intravenous iron over traditional oral iron formulations. A new formulation of oral sucrosomial iron featuring an increased bioavailability compared to traditional oral formulations has recently become available and could provide a valid alternative to those by intravenous (IV) route. Our study evaluated the performance of sucrosomial iron versus intravenous iron in
\end{abstract}

Electronic supplementary material The online version of this article (doi:10.1007/s00520-017-3690-z) contains supplementary material, which is available to authorized users.

Antonino Mafodda

antoninomafodda@gmail.com

D. Giuffrida

giuffridadario@alice.it

A. Prestifilippo

angela.prestifilippo@hotmail.it

D. Azzarello

nicoazzarello@gmail.com

R. Giannicola

roccogiannicola@gmail.com

R. Maisano

robertomaisano@alice.it

1 Department of Oncology, A.O. Bianchi-Melacrino-Morelli, Via Melacrino Giuseppe, 21, 89124 Reggio Calabria, (RC), Italy

2 Istituto Oncologico del Mediterraneo, Via Penninazzo, 7 - 95029, Viagrande, (Catania), Italy increasing hemoglobin in anemic cancer patients receiving chemotherapy and darbepoetin alfa, as well as safety, need of transfusion, and quality of life (QoL).

Materials and methods The present study considered a cohort of 64 patients with chemotherapy-related anemia $(\mathrm{Hb}>8 \mathrm{~g} /$ $\mathrm{dL}<10 \mathrm{~g} / \mathrm{dL}$ ) and no absolute or functional iron deficiency, scheduled to receive chemotherapy and darbepoetin. All patients received darbepoetin alfa $500 \mathrm{mcg}$ once every 3 weeks and were randomly assigned to receive 8 weeks of IV ferric gluconate $125 \mathrm{mg}$ weekly or oral sucrosomial iron $30 \mathrm{mg}$ daily. The primary endpoint was to demonstrate the performance of oral sucrosomial iron in improving $\mathrm{Hb}$ response, compared to intravenous iron. The $\mathrm{Hb}$ response was defined as the $\mathrm{Hb}$ increase $\geq 2 \mathrm{~g} / \mathrm{dL}$ from baseline or the attainment $\mathrm{Hb} \geq 12 \mathrm{~g} / \mathrm{dL}$. Results There was no difference in the $\mathrm{Hb}$ response rate between the two treatment arms. Seventy one percent of patients treated with IV iron achieved an erythropoietic response, compared to $70 \%$ of patients treated with oral iron. By conventional criteria, this difference is considered to be not statistically significant. There were also no differences in the proportion of patients requiring red blood cell transfusions and changes in QoL. Sucrosomial oral iron was better tolerated. Conclusion In cancer patients with chemotherapy-related anemia receiving darbepoetin alfa, sucrosomial oral iron provides similar increase in $\mathrm{Hb}$ levels and $\mathrm{Hb}$ response, with higher tolerability without the risks or side effects of IV iron.

Keywords Chemotherapy-related anemia · Oral iron · IV iron $\cdot \mathrm{Hb}$ response $\cdot$ Sucrosomial iron

\section{Introduction}

Cancer-associated anemia is a common condition reflecting a pathologic deficiency in the amount of oxygen-carrying 
hemoglobin in red blood cells (RBC). It is estimated that the prevalence of anemia in cancer patients before starting cancer treatment is approximately $40 \%$ (due to the disease itself, blood loss, hemolysis, bone marrow infiltration, etc.), with incidence rising to over $80 \%$ as a consequence of chemotherapy (myelosuppression by chemo- and radiotherapy, toxicity of anti-targeted therapies) [1,2]. Furthermore, cancer patients may present an absolute iron deficiency (AID) due to bleeding or poor iron diet or a functional iron deficiency (FID) leading to anemia of chronic disease (ACD), likely caused by a chronic state of inflammation [3-5]. Together, these factors contribute to low hemoglobin $(\mathrm{Hb})$ levels and consequently affect the patient's physical performance and QoL, disease response, and disease morbidity [5-8].

In accordance with the recommendations by the guidelines of major medical societies [9-12], current treatment for chemotherapy-associated anemia often relies on the use of erythropoietic-stimulating agents (ESAs) to reduce need of transfusion, increase $\mathrm{Hb}$ levels, and relieve anemia-related symptoms; yet response rates to ESA alone have been suboptimal, yielding a positive response in only $40 \%$ of patients [ 4 , 5]. Several major studies [4, 13-15] have demonstrated, however, that better responses in terms of increased $\mathrm{Hb}$ response and decreased ESA treatment time are obtained when ESA is given in combination with iron supplementation, which seems to contribute to the correction of erythropoiesis and to compensation in case of a concomitant iron deficiency $[13,16]$.

To date, iron supplementation in combination with ESAs has demonstrated to be more effective through intravenous infusion (IV), compared to conventional oral iron supplementation $[13,17]$, which would be expected in light of the recent findings on hepcidin and the limited gastrointestinal absorption of iron. According to the study by Auerbach et al. [13] on cancer patients with anemia caused by iron deficiency, the percentage of response to erythropoietin (EPO) was increased by both IV and oral iron supplementation (IV $68 \%$ vs oral $36 \%$ ). The study by Henry et al. on cancer patients undergoing chemotherapy evaluated the effect of IV and oral iron sulfate on patients with and without iron deficiency and found the efficacy of conventional oral supplementation to be markedly lower than IV iron [14]. In the attempt of summarizing findings of published studies, a meta-analysis by Pedrazzoli et al. confirmed the efficacy of IV iron supplementation, in anemic cancer patients, in yielding best hematopoietic response with a quicker time of response and a reduction in the number of blood transfusions [4]. Further insight provided by the review by Karlsson on over six clinical trials evaluating ESAs plus IV iron in cancer patients confirmed findings of previous studies, where erythropoietic response in anemic patients receiving ESA is augmented as result of IV iron supplementation [18]. Finally, it appears that hematopoietic response would be related to the dose of IV iron independent of the patients' iron status $[17,19]$.
However, during the same years of these studies, the Italian Medicines Agency (AIFA) in agreement with the European Medicines Agency (EMA) released a recommendation warning on the risk of potentially fatal hyper-sensitization reactions caused by IV iron, which brought new focus onto other more effective and safer ways of iron supplementation [20,21].

Recently, a new oral formulation of ferric pyrophosphate (Sideral ${ }^{\circledR}$ Forte), featuring high bioavailability (approximately threefold that of the gold standard, ferrous sulfate) and a good safety profile, has become available in most European countries $[22,23]$. The distinguishing feature of this formulation has been assessed in a number of studies [22, 24-27] and has shown to be devoid of common side effects of conventional oral iron supplementation such as stomach pain, nausea, constipation, discoloration of the mucous, and feces. Moreover, its safety has been recently confirmed in a recent randomized study on a cohort of non-anemic pregnant women [26].

In the past 5 years, a number of studies have been focusing on patients with FID or AID; however, they do not consider those patients with cancer-related anemia, who have neither FID nor AID, but who still might benefit from iron supplementation. A previous study on patients with chemotherapyrelated anemia and no iron deficiency has evaluated whether this patient population responded better to darbepoetin (DA) or ESA treatment with IV iron supplementation and found that iron supplementation did significantly reduce treatment failures with DA without causing additional toxicity [15]. Hence, our interest focused on oral sucrosomial iron supplementation on this same type of population.

The primary aim of the present study was to perform a retrospective evaluation on the comparability in efficacy of oral sucrosomial iron supplementation in anemic cancer patients featuring neither FID nor AID and receiving darbepoetin, a relatively long-acting ESA, in comparison with IV iron supplementation in the setting of cancer supportive treatments implemented at our hospitals.

Secondary objectives were safety, effect on number of RBC units transfused, and QoL.

\section{Materials and methods}

\section{Study design and patient population}

This was a retrospective pilot study, performed between May 2012 and Nov 2013, involving two Oncology Departments in Southern Italy. The study considered 64 cancer patients with cancer-related anemia scheduled for chemotherapy and treatment with darbepoetin and iron supplementation.

Inclusion criteria were chemotherapy-related anemia, age $\geq 18$ years, diagnosis of major solid tumors (breast cancer and non-small cell lung cancer), $8<\mathrm{Hb}<10 \mathrm{~g} / \mathrm{dL}$, serum ferritin 
$\geq 100 \mathrm{ng} / \mathrm{mL}$, or transferrin saturation $\geq 15 \%$, Eastern Cooperative Oncology Group Performance Score (ECOG PS) $0-2$, life expectancy $\geq 6$ months, at least 8 weeks of residual chemotherapy independent of the iron status and adequate liver and kidney function.

Exclusion criteria were AID (ferritin below the lower limit of the normal range or transferrin saturation $<10 \%$ ), FID (transferrin saturation $<20 \%$ and ferritin $>100 \mu \mathrm{g} / \mathrm{mL}$ ) [15] or iron overload (transferrin saturation $\geq 45 \%$ and ferritin $\geq 800 \mathrm{ng} / \mathrm{mL}$ ) upon enrolment, primary hematologic disorder causing moderate to severe anemia, bone marrow diseases, known bone marrow involvement, comorbid cardiovascular disease incompatible with ESA treatment, active infections, and ESA treatment or blood transfusions within 8 weeks.

The total observation time was 8 weeks of treatment plus 4 weeks of follow-up during which $\mathrm{Hb}$ and biochemical iron levels were also monitored during weekly checkup.

\section{Treatment}

Patients receiving treatment with darbepoetin alfa $(500 \mathrm{mcg})$ every 3 weeks (as by treatment schedule previously established by the oncologist) were administered iron supplementation, either as IV ferric gluconate or as oral sucrosomial iron (Sideral@ Forte, PharmaNutra SpA, Pisa, Italy), as established by the oncologist for a total of 8 weeks. Sucrosomial iron is a patented formulation made of ferric pyrophosphate, lecithin, and sucrester. Patients receiving IV iron were administered iron by dosages of $125 \mathrm{mg} /$ week of ferric gluconate (Ferlixit, Sanofi), corresponding to two vials diluted in $250 \mathrm{~mL}$ of sodium chloride $0.9 \%$ in $60 \mathrm{~min}$ administered in the out-patient clinic of the oncology center (with emergency personnel, as required by AIFA and EMA recommendation warnings on patient safety) $[20,21]$, whereas patients receiving oral iron were given a dosage of $30 \mathrm{mg} /$ day of sucrosomial iron (Sideral ${ }^{\circledR}$ Forte), corresponding to one capsule/day.

Adjustments to ESA doses were made, as appropriate, based on patient response and clinical status and in agreement with product information sheet [28]: when levels of $\mathrm{Hb}$ were $>12 \mathrm{~g} / \mathrm{dL}$, the dose of ESA was reduced by $25-50 \%$; when $\mathrm{Hb}$ were $>13 \mathrm{~g} / \mathrm{dL}$, treatment with darbepoetin was temporarily interrupted and resumed; in case of a drop of $\mathrm{Hb}$ values below $12 \mathrm{~g} / \mathrm{dL}$, treatment was resumed with a $25 \%$ ESA dose reduction. In the case the $\mathrm{Hb}$ increase was $>2 \mathrm{~g} / \mathrm{dL}$ in 4 weeks, dose was reduced $25-50 \%$.

Patients receiving IV or oral iron had consented to supplementation based on the oncologist's treatment suggestions and recommendations, as they had previously did for all other treatments. The study was conducted in line with principles outlined in the Declaration of Helsinki.

\section{Endpoints and measurements}

The endpoints measured included increase in $\mathrm{Hb}$ levels after administration of oral sucrosomial iron, compared to $\mathrm{Hb}$ levels after administration of IV ferric gluconate. Hb response was defined as $\mathrm{Hb}$ increase $>2 \mathrm{~g} / \mathrm{dL}$ from baseline or achieving $\mathrm{Hb} \geq 12 \mathrm{~g} / \mathrm{dL}$.

Secondary endpoints included parameters referring to safety profile evaluated as occurrence of adverse events (AEs) or serious adverse events (SAEs), need for transfusions (yes/no), patient response time and improvement in QoL scores.

QoL was measured by the use of standard questionnaires validated for evaluating QoL in chronic illness, namely the Functional Assessment of Cancer Therapy for anemia in cancer patients (FACT-An version 4) and the Linear Analog Self Assessment (LASA) for cancer patients during chemotherapy $[29,30]$. Both questionnaires are patient-reported outcome tools covering functional, physical, and emotional areas, and ask patients to provide answer based on a Likert scale. Questionnaires were administered to all patients at baseline and after 8 weeks.

Patient compliance to iron supplementation was monitored during checkup by the oncologist through the patient's reports and/or by patient's request for refill of the empty blisters.

\section{Statistical methods}

Variables were expressed as mean \pm standard deviation or count and proportion, depending on their distributions. Accordingly, comparisons were performed with unpaired samples $t$ test or chi-squared test with continuity correction. Response curves were compared with two-way ANOVA. Computations were executed in SPSS Statistics (IBM), version 21 . A value of $p<0.05$ was considered statistically significant.

\section{Results}

The total patient population considered for the analyses consisted in 64 patients (Table 1) either treated with IV iron or oral iron.

The mean baseline level of measured $\mathrm{Hb}$ was $9.4 \mathrm{~g} / \mathrm{dL}$ in the darbepoetin + sucrosomial iron group and $9.2 \mathrm{~g} / \mathrm{dL}$ in the darbepoetin + IV iron group. At least $30 \%$ of patients in each treatment group were treated with platinum-based chemotherapy, a therapy that heavily influences treatment-induced anemia compared to other types of chemotherapy.

Of all patients recruited and undergoing treatment, there were no drop-outs or cases of non- compliance to iron supplementation. 
Table 1 Patient baseline characteristics $(n=64)$

\begin{tabular}{|c|c|c|c|}
\hline & $\begin{array}{l}\text { Darbepoetin }+ \\
\text { sucrosomial } \\
\text { iron } \\
n=33\end{array}$ & $\begin{array}{l}\text { Darbepoetin }+ \\
\text { IV iron } \\
n=31\end{array}$ & $p$ \\
\hline \multicolumn{4}{|l|}{ Sex } \\
\hline Female, $n(\%)$ & $13(40)$ & $12(39)$ & 1 \\
\hline Males, $n(\%)$ & $20(60)$ & $19(61)$ & 1 \\
\hline \multicolumn{4}{|l|}{ Type of cancer } \\
\hline Breast, $n(\%)$ & $10(30)$ & $10(32)$ & 1 \\
\hline Lung, $n(\%)$ & $23(70)$ & $21(68)$ & 1 \\
\hline \multicolumn{4}{|l|}{ Type of chemotherapy } \\
\hline Chemo platinum, $n(\%)$ & $10(30)$ & $12(38)$ & 1 \\
\hline \multicolumn{4}{|l|}{ ECOG Performance status } \\
\hline $0, n(\%)$ & $22(66)$ & $19(62)$ & 1 \\
\hline $1, n(\%)$ & $10(30)$ & $10(32)$ & 1 \\
\hline $2, n(\%)$ & $1(3)$ & $2(6)$ & 1 \\
\hline \multicolumn{4}{|l|}{ Iron status } \\
\hline Baseline $\mathrm{Hb} \mathrm{g} / \mathrm{dL}$ (mean) & 9.4 & 9.2 & 0.932 \\
\hline
\end{tabular}

\section{Efficacy}

Efficacy analyses have shown that response to treatment (increase in $\mathrm{Hb}>2 \mathrm{~g} / \mathrm{dL}$ from baseline or achieving $\mathrm{Hb} \geq 12 \mathrm{~g} / \mathrm{dL}$ ) was achieved by $71 \%$ of patients treated with IV iron and by $70 \%$ of patients supplemented with oral sucrosomial iron, with no statistically significant differences (Fig. 1). Chisquared was equal to 0.014 with 1 degree of freedom. The two-tailed $p$ value was equal to 0.9060 .

These results support the comparability of oral sucrosomial iron supplementation versus intravenous iron in patients with chemotherapy-related anemia.

\section{Safety}

No differences were found between the two treatment groups as to patients requiring $\mathrm{RBC}$ transfusion. As to adverse or serious adverse events related to iron supplementation, these were found in 6\% of patients in the IV group (adverse reaction due to IV infusion) while none were found in the group supplemented with oral iron, as shown in Table 2.

Overall, $43.7 \%$ of patients reported adverse events. $4.7 \%$ were serious adverse events such as gastrointestinal toxicity (abdominal pain, diarrhea) related to chemotherapy treatment (one patient in Sideral group), infusion reaction to IV iron (two patients in IV iron group), and two thromboembolic events (one per study group) most likely caused by treatment with darbepoetin. One fatal event per treatment group was reported but was not related to iron therapy.

\section{Time to response}

Statistical analysis of the time to maximum response showed a comparable profile between the two treatments $(p=0.902)$ in terms of $\mathrm{Hb}$ increase, as reported in Fig. 2.

\section{RBC transfusions}

The number of transfusions needed was equal in the two groups (one patient in each group, $3 \%$ ).

\section{QoL}

Results from the questionnaires in both patient groups showed comparable changes in quality of life parameters and compatible with a gain in $\mathrm{Hb}$ levels. In particular, items most specifically linked to benefits from iron (fatigue/tiredness, general weakness, shortness of breath, feeling energetic, function, being too tired to eat and motivation to perform daily chores) confirmed an improved QoL perception in both groups (Supplemental material).

\section{Discussion}

Despite it is acknowledged that the use of iron supplementation in addition to ESA treatment significantly improves the hematopoietic response to ESAs in dialysis patients with chronic anemia, the use of oral supplementation in cancer patients is still suboptimal [4]. In chronic illnesses, the establishment of a permanent inflammatory status leads to the production of cytokines such as IL- $1 \alpha$ and TNF- $\alpha$, that prevents EPO production from kidneys, and the pro-inflammatory cytokine IL- 6 that acts on the liver and induces the production of hepcidin. This peptide plays a key role in iron homeostasis, inhibiting intestinal iron absorption and impeding the release of iron from the stores [2, 31-33]. The presence of inflammation and, thus,

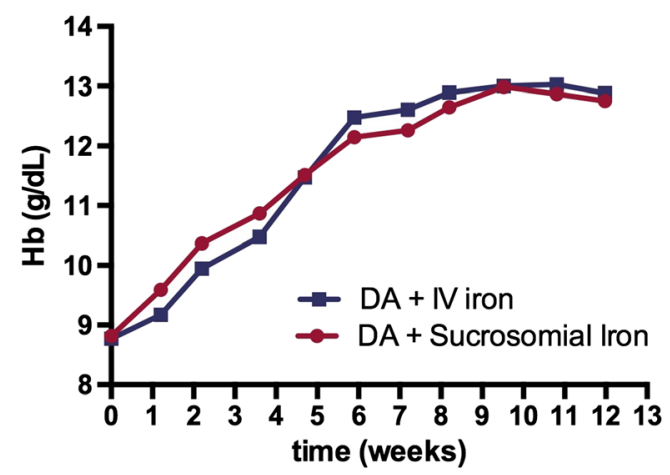

Fig. $1 \mathrm{Hb}$ response in the two treatment groups 
Table 2 Adverse events (AEs) and serious AEs reported in patients by treatment groups

\begin{tabular}{llll}
\hline & $\begin{array}{l}\text { Darbepoetin }+ \\
\text { sucrosomial iron } n=33\end{array}$ & $\begin{array}{l}\text { Darbepoetin }+ \\
\text { IV iron } n=31\end{array}$ & $p$ \\
\hline Patients with adverse events, $n(\%)$ & $13(39)$ & $15(48)$ & 0.636 \\
Patients with serious adverse events, $n(\%)$ & $2(6)$ & $1(3)$ & 1 \\
Gastrointestinal toxicity EPS-related, $n(\%)$ & $1(3)$ & 0 & 1 \\
Infusion reaction to IV iron, $n(\%)$ & 0 & $2(6)$ & 1 \\
Thromboembolic events, $n(\%)$ & $1(3)$ & $1(3)$ & 1 \\
Fatal events & $1(3)$ & $1(3)$ & 1 \\
$\quad$ Overall, $n(\%)$ & 0 & 0 & 1 \\
$\quad$ Treatment related, $n(\%)$ & & & \\
\hline
\end{tabular}

high levels of hepcidin may limit the availability of circulating iron needed by $\mathrm{RBC}$ progenitors, hindering a correct erythropoiesis and eventually leading to the development of anemia of chronic disease [5, 34]. Thus, cancer patients, who present high or normal ferritin level, but also low circulating iron levels, may benefit from iron supplementation.

The issue of chemotherapy-induced anemia and low $\mathrm{Hb}$ levels in cancer patients is associated with poor physical performance status and disease-related fatigue.

Until recently (with the understanding of the mechanisms underlying FID), anemia has been largely underestimated. In 2004, an ECAM survey documented that anemia was left untreated in over $61 \%$ of cancer patients, being partly explained by a broad misconception around anemia in cancer patients (who often feature normal serum ferritin levels) and around the poor tolerability towards traditionally used iron formulations [3, 4], which is no longer applicable to newer formulations.

As a result of a growing number of studies on iron supplementation with ESAs [5], recent guidelines on the use of these agents [11] stress the importance of monitoring iron levels in patients and providing adequate supplementation in order for the patient to gain more benefit from ESA treatment, to improve $\mathrm{Hb}$ response, and to restore functional iron levels.

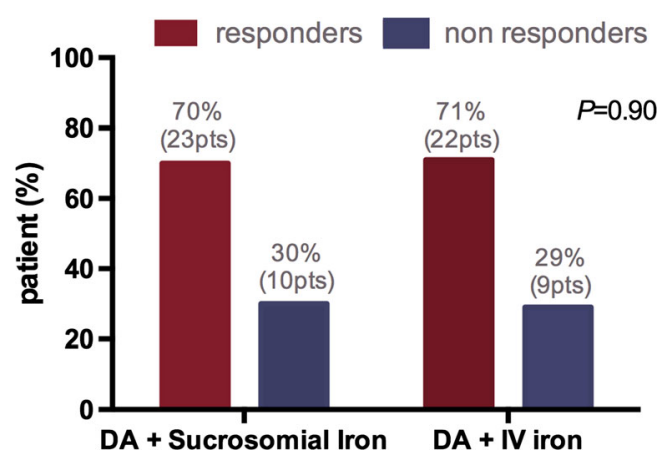

Fig. 2 Comparison of time to response (in weeks) between the two treatment groups
The present study specifically addressed the comparability of oral sucrosomial iron to ferric gluconate, a broadly used IV formulation, which has already been evaluated in a number of previous studies $[14,15]$ in combination with the long-acting ESA, darbepoetin.

Sucrosomial iron (Sideral $®$ ) is a preparation of ferric pyrophosphate conveyed and protected into a phospholipids and sucrester matrix. Differently from other formulations, it features low non-toxic dose of sucresters, which protect iron from the acid environment of the stomach and increase its permeability, allowing higher absorption of exogenous iron [22, 23]. In addition, it is characterized by an alternative route of absorption and delivery, which allows the reduction of side effects and the prevention of iron instability in the gastrointestinal tract. Preliminary studies seem to suggest that sucrosomial iron might also exert a down-regulatory effect on hepcidin in chronic inflammation [35]. Taken together, these characteristics provide obvious advantages over other oral formulations, which have resulted less efficacious, compared to IV iron supplementation, in supporting $\mathrm{Hb}$ response in cancer patients receiving treatment with ESA.

Overall results showed comparable efficacy of sucrosomial iron to that of ferric gluconate in anemic patients not FID and not AID. Both IV and oral sucrosomial iron gave a good response rate (70 and $71 \%$, respectively), with a maximum response rate achieved among patients with $\mathrm{Hb}>9 \mathrm{~g} / \mathrm{dL}$ and a significantly lower response rate among patients with $\mathrm{Hb}$ $\leq 9 \mathrm{~g} / \mathrm{dL}$. This could be explained by the delicate balance involved in iron homeostasis.

In reference to the time to response, oral sucrosomial iron showed a comparable result to IV iron that was maintained for the rest of the monitoring period. Time to $\mathrm{Hb}$ response is an important parameter to consider in cancer patients undergoing treatment with ESA. As it is well demonstrated, ESAs are associated to severe AEs (venous thromboembolism, tumor progression, stroke, and recurrence of disease) and overall mortality [36-38]. In fact, recent guidelines such as $\mathrm{ASCO}$ and $\mathrm{NCCN}[10,11]$ 
recommend that ESA should be administered for the least amount of time and at the minimum dose possible to elicit sufficient RBC levels to limit the need to turn to blood transfusions and to improve QoL. A quicker response, thus, is desirable.

An observational study by Giordano et al. [39] on sucrosomial iron in patients with sideropenic anemia confirmed the reduction of the typical side effects of a conventional martial treatment, determining at the same time an improvement of the patient's anemic condition in terms of serum iron, $\mathrm{Hb}$, and ferritin [24, 25, 39]. Another study by Pisani et al. [27] in patients with chronic kidney disease demonstrated the non-inferiority of the oral sucrosomial iron compared to IV iron therapy in terms of increase in $\mathrm{Hb}$ levels.

From the QoL perspective, iron supplementations represent a remarkable aid in dealing with cancer-related fatigue and preventing other related symptoms such as impaired cognitive function, headache, dizziness, chest pain, shortness of breath, nausea, and depression $[6,29,30]$. A recent German study on the overall QoL, as measured by the FACT-An total scores, showed a median anemiarelated score improvement for patients receiving IV iron or ESA associated with IV iron after 12 weeks of treatment. The difference reached the level of clinical relevance $(\geq 7$ points) for patients receiving ESA associated with IV iron (106.5 to 117.5 points) [40]. Likewise, the same tool, which is specifically validated for assessment in anemic cancer patients, was used also for our cohort.

All patients (both in the group of oral and IV supplementation) equally reported perceived benefits at 8 weeks. Parameters, such as fatigue/tiredness, general weakness, shortness of breath, feeling energetic, function, being too tired to eat, and motivation to perform daily chores, were all improved as expected with a gain in $\mathrm{Hb}$ level. Despite the small study population, such improvement quality of life represents a great achievement in patients with noncurable cancer, as it allows individuals to maintain function in daily life activities (in many cases these may also extend to family duties, work, etc.). The lack of differences in responses between the two groups is encouraging and confirms a comparable effect to IV as to benefits on QoL. Moreover, comparing the safety profile and efficacy of iron supplementations, the option of an effective oral supplementation in capsules may be less burdensome on the patient's logistics and further improve the patient's QoL, as compared to a more invasive IV iron infusion at the outpatient clinic.

From a safety point of view, the advantages of oral sucrosomial iron supplementation are even more relevant in consideration of the recent safety warning $[20,21]$ on the potentially fatal effects of IV iron infusions, making an oral iron supplementation even more desirable.
From the prospective of patient management and costs involved for the healthcare system, IV infusion has also specific procedure requirements. Firstly, each IV session requires that the hematology unit reserve a transfusion station/seat for the time required for the procedure, monitor the patient (approximately $3 \mathrm{~h}$ ), and dedicate personnel on shift during operation, which occupies resources that could be dedicated to other duties. Considering that the need for IV iron supplementation is evaluated on a patient-to-patient basis, the frequency of supplementation might imply up to three infusions per week. Secondly, IV iron can only be performed in authorized centers equipped with emergency and intensive care professionals, which excludes many patient reference centers at local level, thus, potentially discouraging physicians and patients from supplementation.

Overall, performance of oral sucrosomial iron was comparable to that of IV iron and produced comparable advantages for patients in both functional and emotional wellbeing, and $\mathrm{Hb}$ levels, suggesting a potential benefit also in reducing the need for transfusions for such patients and the morbidity of the malignancy.

\section{Limits}

The study was performed on a small sample size and was based on data from only two oncology centers, which limits the generalizability of the results.

Despite presenting an important bias given by the choice of treating both patients with iron deficit and true-replete patients with $\mathrm{Hb}>9 \mathrm{~g} / \mathrm{dL}$, the study provided important findings on the efficacy and safety of oral sucrosomial iron in patients treated with ESA.

Taken together, our study represents a small contribution in addressing the objectives set by ASCO (and other societies thereafter) since 2007, requesting further research to be done on iron supplementation in cancer patients treated with ESA and on iron formulation.

\section{Conclusions}

Oral sucrosomial iron supplementation in association to darbepoetin has shown comparable efficacy to IV ferric gluconate associated to ESA in the treatment of chemotherapyrelated anemia. Moreover, in consideration of the recent safety warning on IV iron and its potential serious adverse events, oral sucrosomial iron represents a valid alternative to the more invasive IV iron supplementation.

The sophisticated sucrosomial technology allows a higher absorption and bioavailability of iron with minimal side effects on the gastrointestinal tract. 
Acknowledgements The Authors are grateful to Manuella Walker (Pencil and Papers, Italy) for the editing of the manuscript.

\section{Compliance with ethical standards}

Conflict of interest The principal investigator received honoraria/ travel expense reimbursement by PharmaNutra (Italy) for his participation as speaker during a CME event. All data are available upon request.

Open Access This article is distributed under the terms of the Creative Commons Attribution-NonCommercial 4.0 International License (http:// creativecommons.org/licenses/by-nc/4.0/), which permits any noncommercial use, distribution, and reproduction in any medium, provided you give appropriate credit to the original author(s) and the source, provide a link to the Creative Commons license, and indicate if changes were made.

\section{References}

1. Dicato M, Plawny L, Diederich M (2010) Anemia in cancer. Ann Oncol 21(Suppl 7):vii167-vii172

2. Gascon P (2015) Management of iron-deficiency anemia and functional iron deficiency in cancer patients. Expert Rev Hematology, Rome, 8(S1):1-32

3. Ludwig H, Muldur E, Endler G, Hubl W (2013) Prevalence of iron deficiency across different tumors and its association with poor performance status, disease status and anemia. Annals of Oncology 24(7):1886-1892

4. Pedrazzoli P, Rosti G, Secondino S, Siena S (2009) Iron supplementation and erythropoiesis-stimulatory agents in the treatment of cancer anemia. Cancer 115(6):1169-1173

5. Steinmetz HT (2012) The role of intravenous iron in the treatment of anemia in cancer patients. Ther Adv Hematol 3(3):177-191

6. Bohlius J, Tonia T, Nuesch E, Juni P, Fey MF, Egger M et al (2014) Effects of erythropoiesis-stimulating agents on fatigue- and anaemia-related symptoms in cancer patients: systematic review and meta-analyses of published and unpublished data. $\mathrm{Br} \mathrm{J}$ Cancer 111(1):33-45

7. Ludwig H, Aapro M, Bokemeyer C, Glaspy J, Hedenus M, Littlewood TJ et al (2014) A European patient record study on diagnosis and treatment of chemotherapy-induced anaemia. Support Care Cancer 22(8):2197-2206

8. Ludwig H, Van Belle S, Barrett-Lee P, Birgegard G, Bokemeyer C, Gascon P et al (2004) The European Cancer Anaemia Survey (ECAS): a large, multinational, prospective survey defining the prevalence, incidence, and treatment of anaemia in cancer patients. Eur J Cancer 40(15):2293-2306

9. Bokemeyer C, Aapro MS, Courdi A, Foubert J, Link H, Osterborg A et al (2007) EORTC guidelines for the use of erythropoietic proteins in anaemic patients with cancer: 2006 update. Eur J Cancer 43(2):258-270

10. Rizzo JD, Brouwers M, Hurley P, Seidenfeld J, Somerfield MR, Temin S (2010) American Society of Clinical Oncology/American Society of Hematology Clinical Practice Guideline update on the use of epoetin and darbepoetin in adult patients with cancer. J Oncol Pract 6(6):317-320

11. Rodgers GM 3rd, Becker PS, Blinder M, Cella D, Chanan-Khan A, Cleeland C et al (2012) Cancer- and chemotherapy-induced anemia. J Natl Compr Canc Netw: JNCCN 10(5):628-653

12. Schrijvers D, De Samblanx H, Roila F (2010) Erythropoiesisstimulating agents in the treatment of anaemia in cancer patients: ESMO Clinical Practice Guidelines for use. Ann Oncol 21(Suppl 5):v244-v247
13. Auerbach M, Ballard H, Trout JR, McIlwain M, Ackerman A, Bahrain $\mathrm{H}$ et al (2004) Intravenous iron optimizes the response to recombinant human erythropoietin in cancer patients with chemotherapy-related anemia: a multicenter, open-label, randomized trial. J Clin Oncol 22(7):1301-1307

14. Henry DH, Dahl NV, Auerbach M, Tchekmedyian S, Laufman LR (2007) Intravenous ferric gluconate significantly improves response to epoetin alfa versus oral iron or no iron in anemic patients with cancer receiving chemotherapy. Oncologist 12(2):231-242

15. Pedrazzoli P, Farris A, Del Prete S, Del Gaizo F, Ferrari D, Bianchessi C et al (2008) Randomized trial of intravenous iron supplementation in patients with chemotherapy-related anemia without iron deficiency treated with darbepoetin alpha. J Clin Oncol 26(10):1619-1625

16. Aapro M, Osterborg A, Gascon P, Ludwig H, Beguin Y (2012) Prevalence and management of cancer-related anaemia, iron deficiency and the specific role of i.V. Iron. Ann Oncol 23(8):1954-1962

17. Petrelli F, Borgonovo K, Cabiddu M, Lonati V, Barni S (2012) Addition of iron to erythropoiesis-stimulating agents in cancer patients: a meta-analysis of randomized trials. J Cancer Res Clin Oncol 138(2):179-187

18. Karlsson T (2011) Effects of iron supplementation on erythropoietic response in patients with cancer-associated anemia treated by means of erythropoietic stimulating agents. ISRN Hematol 2011:108397

19. Gafter-Gvili A, Rozen-Zvi B, Vidal L, Leibovici L, Vansteenkiste J, Gafter U et al (2013) Intravenous iron supplementation for the treatment of chemotherapy-induced anaemia - systematic review and meta-analysis of randomised controlled trials. Acta Oncol (Stockholm, Sweden) 52(1):18-29

20. AIFA (2013) Nota Informativa importante su medicinali contententi ferro per via endovenosa. Available at: http://www.aifa.gov.it/ content/nota-informativa-importante-su-medicinali-contenentiferro-25102013. Accessed 7 April 2017.

21. European Medicine Authority (2013) New recommendations to manage risk of allergic reactions with intravenous iron-containing medicines EMA/579491/2013: EMA [updated 13 September 2013. Available from: http://www.ema.europa.eu/docs/en GB/document library/Referrals_document/IV_iron_31/WC500150771.pdf

22. Brilli E, Romano A, Fabiano A, Zambito Y, Di Raimondo F, Tarantino G (2016) Sucrosomial ${ }^{\circledR}$ technology is able to promote ferric iron absorption: pre-clinical and clinical evidences. Blood J 128(22):3618

23. Tarantino G, Brilli E, Zambito Y, Giordano G, Equitani F (2015) Sucrosomial Iron®: a new highly Bioavaible oral iron supplement. Blood 126(23):4561

24. Barni S (2015) 3rd Mediterranean Multidisciplinary Course on Iron Anemia April, 17(th)-18(th) 2015, Rome, Italy. Expert Rev Hematol 8(Suppl 1):S1-s32

25. Barni S (2016) 4th Mediterranean Multidisciplinary Course on Iron Anemia April 29th-30th 2016, Madrid, Spain. Expert Rev Hematol 9(sup1):1-42

26. Parisi F, Berti C, Mandò C, Martinelli A, Mazzali C, Cetin I (2016) Effects of different regimens of iron prophylaxis on maternal iron status and pregnancy outcome: a randomized control trial. The Journal of Maternal-Fetal \& Neonatal Medicine 1-21

27. Pisani A, Riccio E, Sabbatini M, Andreucci M, Del Rio A, Visciano B (2015) Effect of oral liposomal iron versus intravenous iron for treatment of iron deficiency anaemia in CKD patients: a randomized trial. Nephrol Dial Transplant 30(4):645-652

28. European Medicine Authority (2013) Aranesp product information sheet. [Available from: http://www.ema.europa.eu/docs/en_GB/ document library/EPAR - Summary for the public/human/ 000332/WC̄500026141.pdf.

29. Gunnars B, Nygren P, Glimelius B (2001) Assessment of quality of life during chemotherapy. Acta Oncol (Stockholm, Sweden) 40(23):175-184 
30. Yellen SB, Cella DF, Webster K, Blendowski C, Kaplan E (1997) Measuring fatigue and other anemia-related symptoms with the Functional Assessment of Cancer Therapy (FACT) measurement system. J Pain Symptom Manag 13(2):63-74

31. Nemeth E, Ganz T (2006) Hepcidin and iron-loading anemias. Haematologica 91(6):727-732

32. Nemeth E, Ganz T (2014) Anemia of inflammation. Hematol Oncol Clin N Am 28(4):671-681 vi

33. Roy CN, Andrews NC (2005) Anemia of inflammation: the hepcidin link. Curr Opin Hematol 12(2):107-111

34. Ganz T (2011) Hepcidin and iron regulation, 10 years later. Blood 117(17):4425-4433

35. Tarantino G, Brilli E, Giordano G, Torelli A, Equitani F (2015) Innovative oral iron supplement (Sucrosomial Iron ${ }^{\circledR}$ ) is able to downregulate Hepcidin release during inflammation: in vitro study. Blood 125(23):4563

36. Bohlius J, Schmidlin K, Brillant C, Schwarzer G, Trelle S, Seidenfeld J et al (2009) Recombinant human erythropoiesis- stimulating agents and mortality in patients with cancer: a metaanalysis of randomised trials. Lancet 373(9674):1532-1542

37. Kara O, Dizdar O, Altundag K (2008) Erythropoiesis-stimulating agents in the treatment of cancer-associated anemia. JAMA 300(24):2855 author reply -7

38. Wright JR, Ung YC, Julian JA, Pritchard KI, Whelan TJ, Smith C et al (2007) Randomized, double-blind, placebo-controlled trial of erythropoietin in non-small-cell lung cancer with disease-related anemia. J Clin Oncol 25(9):1027-1032

39. Giordano G, Mondello P, Tambaro R, Perrotta N, D'Amico F, D'Aveta A et al (2015) Biosimilar epoetin $\alpha$ is as effective as originator epoetin- $\alpha$ plus liposomal iron (Sideral ${ }^{\circledR}$ ), vitamin B12 and folates in patients with refractory anemia: a retrospective real-life approach. Mol Clin Oncol 3(4):781-784

40. Steinmetz T, Schroder J, Plath M, Link H, Vogt M, Frank M et al (2016) Antianemic treatment of cancer patients in German routine practice: data from a prospective cohort study-the tumor anemia registry. Anemia 2016:8057650 